INPLASY

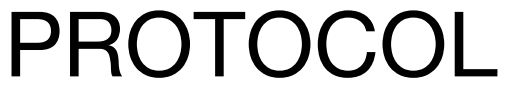

To cite: Tan et al. Chinese herbal medicine for Triple Negative Breast Cancer

patients: A protocol for a systematic review and metaanalysis. Inplasy protocol 202130029. doi:

10.37766/inplasy2021.3.0029

Received: 09 March 2021

Published: 09 March 2021

Corresponding author: Yuanfu QI

m13793132317@163.com

Author Affiliation:

Affiliated Hospital of Shandong

University of Traditional

Chinese Medicine

Support: CMSTDP of

shandong (2019-0091).

Review Stage at time of this submission: Preliminary searches.

Conflicts of interest:

None declared.

\section{Chinese herbal medicine for Triple Negative Breast Cancer patients: A protocol for a systematic review and meta-analysis}

Tan, Z1; Sun, Y²; Song, Y3; Zhang, C4; Fu, R5; Qi, Y6.

Review question / Objective: P: Randomized controlled trials regarding efficacy and safety of TCM in the treatment of TNBC. I: TCM or TCM combined with other treatments (including surgery, radiotherapy, chemotherapy, Chinese medicine, etc.). C: conventional therapies (including surgery, radiotherapy, chemotherapy)alone or combined. O: Primary outcomes: solid tumors and 3-year or 5-year survival rate; secondary outcomes: Progress Free Survival, quality of life evaluated with Karnofsky score, and adverse events. S: Randomized controlled trials regarding efficacy and safety of TCM in the treatment of TNBC will be included without restriction language.

Information sources: We searched the Cochrane Library, PubMed, EMBASE and four Chinese databases, including CNKI, VIP, CBM and Wanfang Data from their date of inception till Dec 31th, 2020.

INPLASY registration number: This protocol was registered with the International Platform of Registered Systematic Review and Meta-Analysis Protocols (INPLASY) on 09 March 2021 and was last updated on 09 March 2021 (registration number INPLASY202130029).

\section{INTRODUCTION}

Review question / Objective: P: Randomized controlled trials regarding efficacy and safety of TCM in the treatment of TNBC. I: TCM or TCM combined with other treatments (including surgery, radiotherapy, chemotherapy, Chinese medicine, etc.). C: conventional therapies (including surgery, radiotherapy, chemotherapy)alone or combined. O: Primary outcomes: solid tumors and 3-year or 5-year survival rate; secondary outcomes: Progress Free Survival, quality 
of life evaluated with Karnofsky score, and adverse events. S: Randomized controlled trials regarding efficacy and safety of TCM in the treatment of TNBC will be included without restriction language.

Condition being studied: In China, Breast carcinoma cases (include TNBC)also use Chinese Herbal Medicine(TCM) during or after receiving chemotherapy. Increasing studies have identified that traditional Chinese medicine and its active ingredients exert a positive therapeutic effect on TNBC. According to several trials in clinics, TCM is available for improving the therapeutic effect of chemotherapy and prolong patient, S OS. TCM is also considered a vital supplementing therapy exerting positive influences on TNBC which can help mitigate chemotherapyassociated side influences, elevate patient survival and life quality. Over the past few years, rising clinical studies that showed TCM efficacy and safety in terms of TNBC received the conduction. However, conclusions of the mentioned trials conducted by complying with comparatively low sizes of samples might lead to conflicts. For the mentioned reason, the present systematically reviewing study was carried out for evaluating TCM efficacy and safety, adjuvant to chemotherapy in terms of cases with TNBC.

\section{METHODS}

Participant or population: No restrict.

Intervention: According to the Pharmacopoeia of the People's Republic of China edited by the China Food and Drug Administration in 2015, TCM was defined as herbal agents and materials that sources. The formulations of TCM included decoction, tablet, pill, powder, granule, capsule, oral liquid, and injection. Usually, a TCM formula is composed of 2 or more herbs to achieve synergistic effect for certain conditions, which is prescribed based on the traditional Chinese medicine pattern diagnosis and treatment thresholds by experienced physicians.
Comparator: Participants in the control group should be treated by conventional therapies (including surgery, radiotherapy, chemotherapy)alone or combined.

Study designs to be included: We define studies as Randomized controlled trials regarding efficacy and safety of TCM in the treatment of TNBC, regardless of whether the blinding method is implemented for subjects, researchers, and observers, and whether the allocation is hidden or not.

Eligibility criteria: We included randomized controlled trials that aim to explore the the efficacy and safety of TCM or TCM combined with other treatments (including surgery, radiotherapy, chemotherapy, Chinese medicine, etc.) for patients with TNBC. This study includes Study registration, Literature retrieval, data analysis, Statistical analysis, registration of protocol, operation, data management, Sensitivity analysis, Dealing with missing data, Assessment of quality, Assessment of heterogeneity and reporting bias, reporting or dissemination of findings. No publication status or language restriction was applied.

Information sources: We searched the Cochrane Library, PubMed, EMBASE and four Chinese databases, including CNKI, VIP, CBM and Wanfang Data from their date of inception till Dec 31th, 2020.

Main outcome(s): Therapeutic effect according to standard for therapeutic effect evaluation of solid tumor by response evaluation criteria in solid tumors and 3-year or 5-year survival rate.b. Progress Free Survival, quality of life evaluated with Karnofsky score, and adverse events.

Quality assessment / Risk of bias analysis: The GRADE (Grades of Recommendations Assessment, Development and Evaluation) system was used to evaluate the quality of each selected studies by 3 investigators, including bias risk, indirect risk, inconsistency risk, imprecise risk and publication bias. 
Strategy of data synthesis: Note Express was used for literature management. We will use Q-squared and 12 tests to evaluate the heterogeneity of all studies included and RevMan 5.3 software to calculate for data synthesis. Sensitivity analysis will be implemented through the R2WinBUGS package of $R$ software.

Subgroup analysis: If the data are abundant, the heterogeneity will be dealt with by subgroup analysis according to sex, locations, histologic diagnosis, TNM stage, duration of TCM therapies, timing of TCM therapies, chemotherapy regimens.

Sensitivity analysis: Sensitivity analysis will be implemented through the R2WinBUGS package of $R$ software to verify the clinical and methodological similarities between the included studies and determine the reliability of this study's results.

Language: Chinese, English.

Country(ies) involved: China, Canada, the United States.

Keywords: triple-negative Breast Cancer, solid tumor, adverse events, Karnofsky, Progress Free Survival, traditional chinese medicine.

Contributions of each author:

Author 1 - Zhaofeng Tan - The author drafted the protocol.

Email: 2018101069@sdutcm.edu.cn

Author 2 - Yuli Sun - The author provided advice for the study design and revised the protocol.

Email: sunyulijn@126.com

Author 3 - Yonglei Song - The author provided advice for the study design and revised the protocol.

Email: isongyonglei@163.com

Author 4 - Chuanlong Zhang - The author provided advice for the study design and revised the protocol.

Email: zcl163yxzh@163.com

Author 5 - Rong Fu - The author provided advice for the study design and revised the protocol.

Email: sdzyfr1916@163.com
Author 6 - Yuanfu Qi - The author drafted the protocol.

Email: m13793132317@163.com 